\title{
Syndrome de Pancoast-Tobias
}

\section{Pancoast-Tobias Syndrome}

\section{Rida-Chafi - C. Touquet $\cdot$ M. Violeau $\cdot$ I. Rafei-Darmian}

Reçu le 21 mars 2013 ; accepté le 2 mai 2013

(C) SFMU et Springer-Verlag France 2013

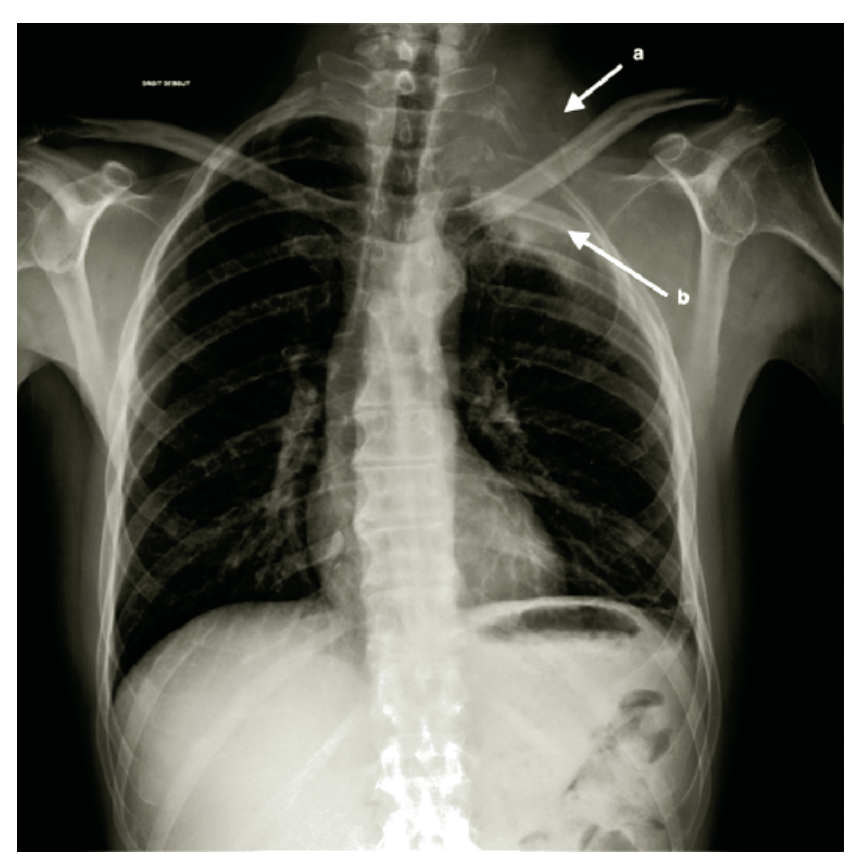

Fig. 1 Radio pulmonaire de face : a : lyse osseuse ; b : lésion tumorale
Un patient de 53 ans est adressé aux urgences pour la majoration d'une névralgie cervicobrachiale C8-T1, attribuée depuis deux ans à une sténose canalaire cervicale objectivée sur une IRM. Il présente une douleur du membre supérieur gauche de topographie C8-T1, sans déficit moteur. Le patient est gaucher, fumeur, et présente également une légère atrophie musculaire de l'épaule gauche, dans un contexte de tendinopathie de la coiffe des rotateurs pour laquelle il est en maladie professionnelle depuis deux ans. Une radiographie pulmonaire demandée à titre systématique (Fig. 1) retrouve une masse au niveau de l'apex pulmonaire gauche ainsi qu'une destruction de l'arc postérieur des deux premières côtes, faisant suspecter un syndrome de Pancoast-Tobias [1].

\section{Référence}

1. Davis G, Knight S (2008) Pancoast tumors. Neurosurg Clin N Am 19:545-57
I. Rida-Chafi · C. Touquet $(\bowtie) \cdot$ M. Violeau $\cdot$ I. Rafei-Darmian Service des urgences Samu-Smur de Niort,

40, avenue Charles de Gaulle, F-79000 Niort, France

e-mail : touquet.cedric@gmail.com 